\title{
Fuzzy higher type information granules from an uncertainty measurement
}

\author{
Mauricio A. Sanchez ${ }^{1} \cdot$ Juan R. Castro $^{1} \cdot$ Oscar Castillo $^{2}$ - Olivia Mendoza ${ }^{1}$. \\ Antonio Rodriguez-Diaz ${ }^{1}$ Patricia Melin ${ }^{2}$
}

Received: 10 August 2016/Accepted: 30 September 2016/Published online: 15 October 2016

(C) Springer International Publishing Switzerland 2016

\begin{abstract}
This paper proposes a new method for the formation of fuzzy higher type granular models. This is accomplished by directly discovering uncertainty from a sample of numerical information. In this case the coefficient of variation is proposed as a heuristic for measuring uncertainty, where a direct relation between this measure and the footprint of uncertainty of an interval type-2 fuzzy membership function is given. Followed by a steepest descent algorithm which is used to calculate interval Sugeno consequents for a fuzzy inference system. Two synthetic and two real datasets are used, measuring the performance by evaluating the output interval coverage of the formed granular models as well as the coefficient of determination which assesses modeling performance.
\end{abstract}

Keywords Granular computing · Higher type information granules · Fuzzy sets · TSK - Interval type-2 - Steepest descent

Oscar Castillo

ocastillo@tectijuana.mx

Mauricio A. Sanchez

mauricio.sanchez@uabc.edu.mx

Juan R. Castro

jrcastror@uabc.edu.mx

Olivia Mendoza

omendoza@uabc.edu.mx

Antonio Rodriguez-Diaz

ardiaz@uabc.edu.mx

Patricia Melin

pmelin@tectijuana.mx

1 Universidad Autonoma de Baja California, Tijuana, Mexico

2 Tijuana Institute of Technology, Tijuana, Mexico

\section{Introduction}

Uncertainty, as it is currently perceived, is still something of a perplexing subject in computer sciences which could be considered doubtful or unknown, in which by nature cannot be directly measured. And although it is directly not known, an approximate of it can be modeled and used, as this can enhance the models in which it is added to. Using uncertainty in a model, its resilience can improve.

Literature on the basic management on uncertainty (Weise and Woger 1993; Klir and Wierman 1999; Yu and Mehrotra 2003; Klir 2005; Chen et al. 2009; Jurado et al. 2015 ) is mainly based on having previous knowledge of the confidence interval around certain measurements, and is usually expressed with the plus-minus symbol " \pm ", e.g., $7.6 \pm 0.03$. Although more advanced constructs exist which permits the description of uncertainty through various definitions, such as fuzzy sets (Zadeh 1965), intuitionistic fuzzy sets (Atanassov 1986), rough sets (Pawlak 1982), shadowed sets (Pedrycz and Vukovich 2002), interval-valued sets (Zadeh 1975), etc in the case of this paper, fuzzy sets are used.

Granular computing (Pedrycz 2007; Pedrycz and Chen $2015 a, b)$ is based on the notion of how the human mind organizes "known information" into certain levels of specificity, and is used as a means for obtaining enhanced models of known data; and with such, take better decisions. It also modifies and adjusts existing information models so as to improve their general performance, as well as improve the interpretability of its information granule representations. In this case, an information granule is a compact, and well-defined, grouping of information which shares similarities. The concept of uncertainty can be integrated into granular computing models via higher type information granules (Pedrycz and Chen 2011; Pedrycz 
2013), such that, apart from also having the characteristics of information granules, their models also integrate an additional attribute, which is uncertainty management.

As for modeling systems with uncertainty, interval type2 fuzzy logic systems (IT2 FLSs) permit uncertainties to be modeled, where these uncertainties are a portrayal of not precisely knowing the location of the membership grade, and as such are depicted by a Footprint Of Uncertainty (FOU) (Mo et al. 2014). An IT2 FLS can either output an interval-valued type-1 fuzzy set or, via a defuzzification process (Starczewski 2008), a crisp value (Castillo and Melin 2012). In the case of IT2 FLSs, a plethora of applications exists (Chen and Lee 2010a, b, c, 2011; Chen and Chang 2011a, b; Chen et al. 2012, 2013a, b; Chen and Wang 2013; Chen and Hong 2014; Cheng et al. 2016; Mendel 2016), such that they have proven useful in many fields, granular computing applications being no exception. Therefore, since IT2 FSs can intrinsically model uncertainty, they were chosen for representing higher type information granules in this paper.

Two contributions are introduced in this paper. First, an association between uncertainty and a measure of dispersion is presented; second, a granular-inspired steepest descent algorithm is also presented which gives more control over the desired output coverage. The platform for the granular model is created by a fuzzy c-means algorithm (Bezdek et al. 1984); afterwards the coefficient of variation is used as a means to calculate the footprint of uncertainty of each individual IT2 FS in the antecedents, and finally, all IT2 Sugeno linear consequent (Ying 2009) parameters are adjusted via a granular-inspired learning algorithm.

This paper is divided into four main sections, the first is a brief introduction to the definition of IT2 FSs as well as its relation to higher type information granules; the following section describes in detail both the premises and the proposed granular method; afterwards, some experimental results are shown and discussed; finally, concluding remarks are given.

\section{Interval type-2 fuzzy sets as a representation of higher type information granules}

Information granules can be represented by many types of modeling formats, such as rough sets (Sai et al. 2005), type-1 fuzzy sets (Zhang et al. 2009), quotient space (Zhang and Zhang 2005), neural networks (Vasilakos and Stathakis 2004), etc. But these formats cannot properly represent higher type information granules, since they cannot directly manage uncertainty within their models. For this reason, a representation is needed which can handle uncertainty; this requirement can be fulfilled, amongst other representations, by IT2 FSs.
An interval type-2 fuzzy inference system (IT2 FIS) is best described through a block diagram, as shown in Fig. 1. Where the fuzzifier block may or may not transform the crisp input into a fuzzy set (FS), this is chosen depending on the intended behavior of the system; either a singleton or a FS is created. The inference block continues from the rules block and reasons based on each input's compatibility. The type-reducer block processes the obtained outputs into a type-reduced fuzzy set. Finally, the defuzzifier block reduces the type-reduced fuzzy set from the previous block and obtains a crisp value.

In an IT2 FS $\tilde{A}$ is represented by $\underline{\mu}_{\tilde{A}}(x)$ and $\bar{\mu}_{\tilde{A}}(x)$ which are the lower and upper membership functions, respectively, of $\mu_{\tilde{A}}(x)$, expressed as $\tilde{A}=\left\{\left((x, u), \mu_{\tilde{A}}(x, u)=1\right)\right.$ $\forall x \in X, \forall u \in[0,1]\}$, where $x$ is a subset of the universe of discourse $X$, and $u$ is a mapping of $X$ into [0,1]. A sample IT2 FS is shown in Fig. 2, where the gray area represents the uncertainty contained within the FS.

Rule representation for an IT2 FIS is shown in Eq. 1, where, $l=1, \ldots, M$ rules, $p=1, \ldots, q$ inputs, $\tilde{F}$ is an antecedent IT2 FS, and $\tilde{G}$ a consequent IT2 FS.

$R^{l}:$ IF $x_{1}$ is $\tilde{F}_{1}^{l}$ and. . and $x_{p}$ is $\tilde{F}_{p}^{l}$, THEN $y$ is $\tilde{G}^{l}$.

\section{Method for the formation of higher type information granule models}

\subsection{Dispersion in data}

We can start with the premise that uncertainty can be interpreted as a case of data dispersion in a sample of data. An example of varying degrees of dispersion is shown in Fig. 3, where different degrees of dispersion can be perceived: low, medium, and high data dispersion. Each in relation to its center, marked in red.

With low dispersion (Fig. 3a), all data samples near the center are very compact and close to one another; this also causes the standard deviation to be very small which can be interpreted directly as uncertainty in data; having low dispersion means having low uncertainty because its numerical evidence suggests that there is near zero possibility that further samples will fall far from the central point. For the case of medium data dispersion (Fig. 3b), although there is a concentration of numerical evidence near its central point, data points still exist far from its center. This leads to imply that although future acquisition of data might lead to obtain evidence which is far from the center, the probabilities of this occurring are low when compared to having future samples fall near the center. This behavior leads to the interpretation of having a medium amount of uncertainty. With high dispersion case 
Fig. 1 Block diagram describing an IT2 FLS

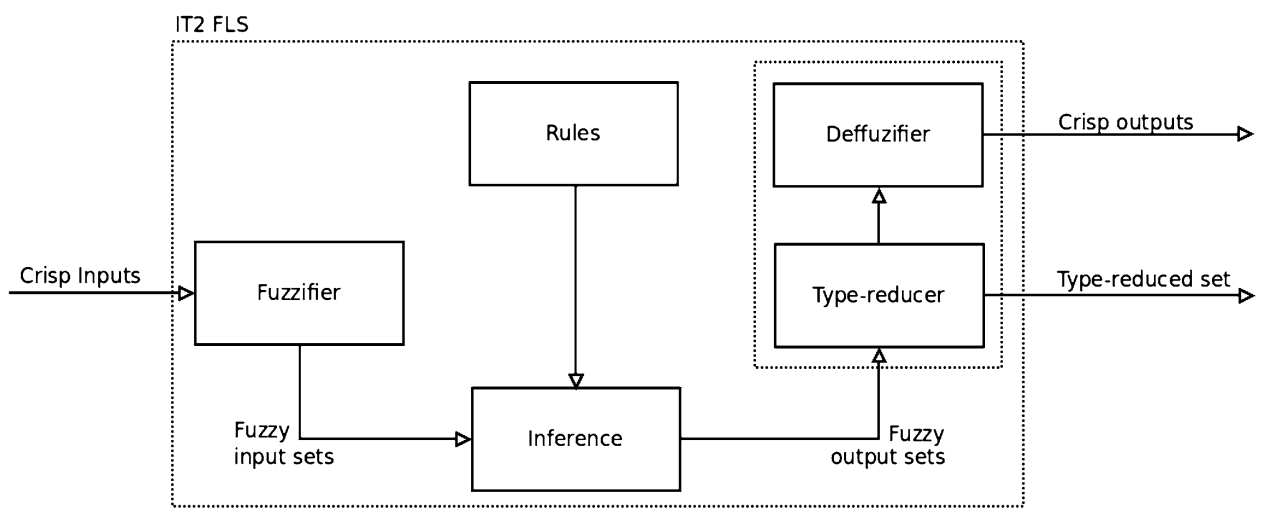

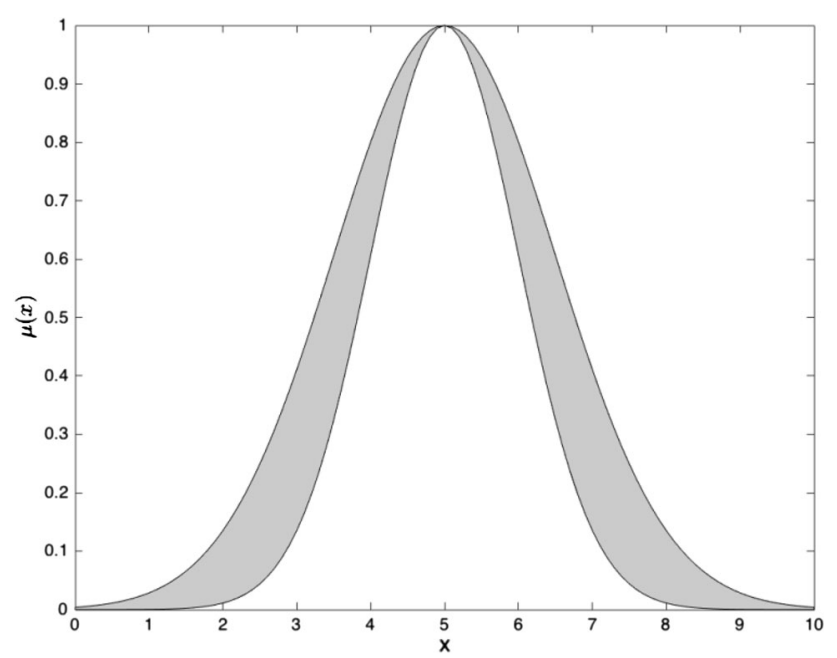

Fig. 2 IT2 Gaussian membership function with uncertainty in the standard deviation

(Fig. 3c), where every sampled data point is evenly distributed throughout the range of possible measurements, the available numerical evidence suggests that any future sample may equally land on any section within the range of the universe of discourse; consequently a high amount of uncertainty exists.

\subsection{Relation of coefficient of variation with uncertainty}

To convert a measure of dispersion into a fuzzy measure of uncertainty, a degree of dispersion in a given set of sample data must first be identified. Such requirement can be obtained using the coefficient of variation $\mathrm{cv}$, shown in Eq. 2, where $\sigma$ is the standard deviation, and $m$ is the mean of the set.

$\mathrm{cv}=\frac{\sigma}{m}$.

This measure has one limitation which undoubtedly must be considered when used in the proposed method. Such limitation is that cv can only be computed on nonnegative values. Although, this only affects the selection of which datasets can be used.

To express the relation of dispersion-uncertainty when dealing with IT2 FSs, the footprint of uncertainty (FOU) is used. Where the FOU of an IT2 FS is an area within bounds covering the primary membership function of $x \in X$, represented by $\underline{\mu}_{\tilde{A}}(x)=\left[\underline{\mu}_{\tilde{A}}(x), \bar{\mu}_{\tilde{A}}(x)\right]$, such that a larger area represents higher uncertainty while a smaller area represents less uncertainty. This relation is a direct proportional

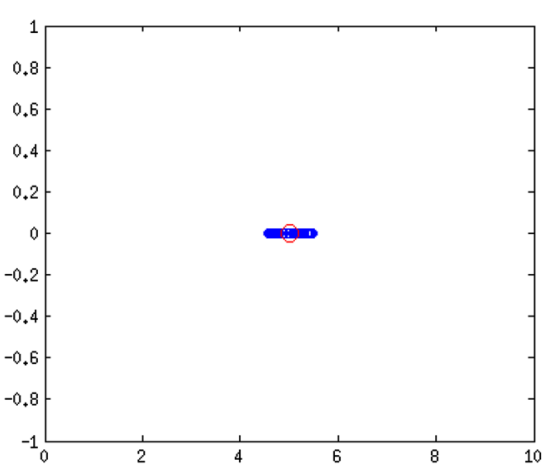

(a)

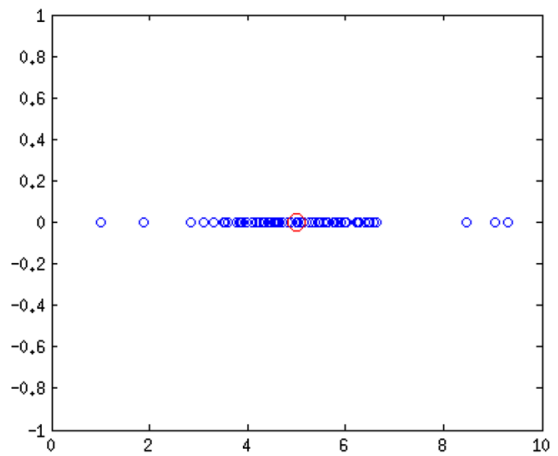

(b)

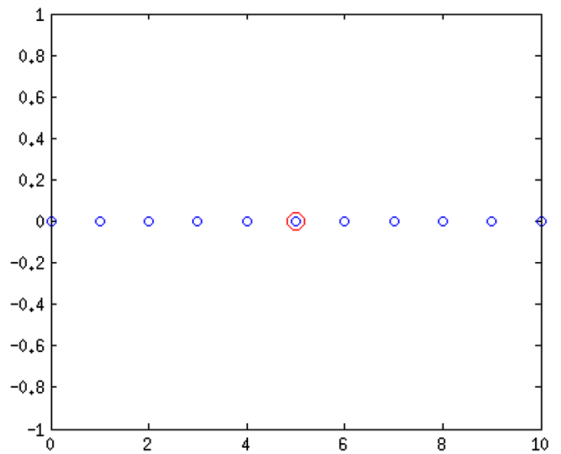

(c)

Fig. 3 Example of data dispersion. a Low data dispersion, b medium data dispersion, and $\mathbf{c}$ high data dispersion 
$\mathrm{cv} \propto$ FOU equivalence. With low dispersion, a small FOU exists, with a medium amount of dispersion, a medium amount of FOU exists, and with a high amount of dispersion, a high amount of FOU exists. This explanation can be better perceived in a visual manner, shown in Fig. 4, varying degrees of a measure of dispersion have been converted into a FOU which directly forms an IT2 FS.

\subsection{Proposed granular method for building higher type information granules}

To form higher type information granules for the antecedents of an IT2 fuzzy system, the first step is to obtain a FIS prototype (rule base, antecedent centers for Gaussian membership functions, and subsets of sample data which created each prototype) via a FCM algorithm. Please note that any other membership function can be used, but we chose Gaussian membership functions for their simplicity, such that they can be formed via two parameters. Each FS is dealt with independently of each other for the process of obtaining higher type information granules. Each FS is composed of a center value $m$, and a standard deviation $\sigma$ obtained from the subset of sample data $\delta$. And for each FS, a cv is calculated using Eq. (2). This value is now used to search for a near optimal FOU area in an IT2 FS. The search starts by first considering the highest possible obtainable area $\mathrm{FOU}^{\max }=\int \tilde{A}(x) \mathrm{dx}=1$ with the previously obtained $\sigma$, as shown in Fig. $4 \mathrm{c}$, achieved when $\sigma_{1}=0$ and $\sigma_{2}=2 \sigma$. Here, possible values of FOU are in the interval $[0,1]$. The search is then performed with discrete small steps $\lambda$ until the FOU value which equals $\mathrm{cv}$ is found. The smallest value $\sigma^{0}$ is defined as $\sigma_{1}=\sigma_{2}=\sigma^{0}=\sigma$, as shown in Fig. 4a. Each step $\lambda$ affects $\sigma_{1}$ and $\sigma_{2}$ by a size increment/decrement, as shown in Eq. (3). This is done iteratively while $\left|\sigma_{1}, \sigma_{2}\right| \leq 2 \sigma$, where $\left|\sigma_{1}, \sigma_{2}\right|$ is the Euclidean distance between $\sigma_{1}$ and $\sigma_{2}$,

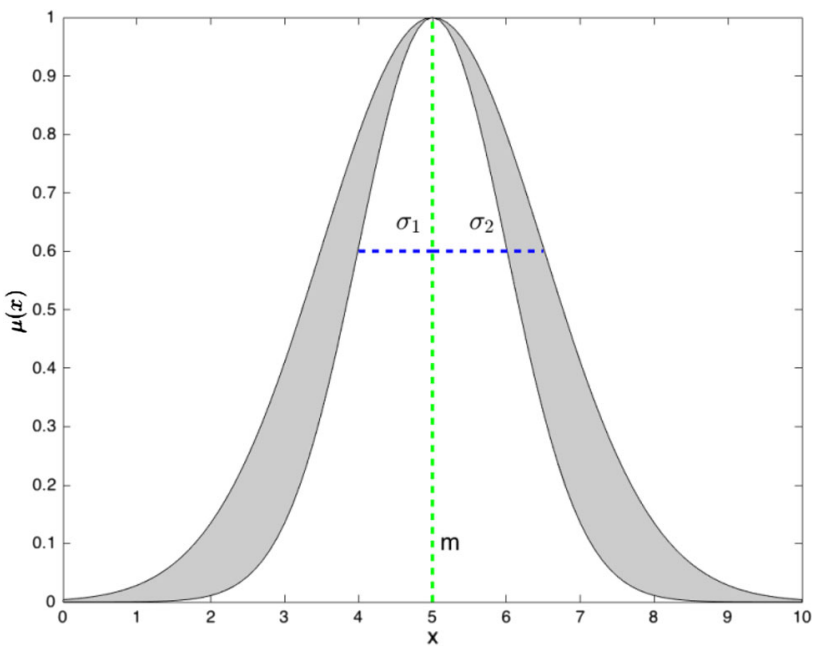

Fig. 5 Higher type information granule represented by an IT2 FS, in the form of a Gaussian membership function with uncertainty in the standard deviation. Characterized by three parameters: $\sigma_{1}, \sigma_{2}$ and $m$

or until $\mathrm{cv}=\mathrm{FOU}_{i}$, and $\mathrm{FOU}_{i}$ is the current iteration of the IT2 FS modified by $\sigma_{1,2}^{i}$.

$\sigma_{1,2}^{i}=\sigma_{1,2}^{i-1} \pm \lambda ; i=0,1, \ldots, n$.

When the search has found the values of $\sigma_{1}$ and $\sigma_{2}$ which together form the desired FOU, the IT2 FS can be formed, i.e., a higher type information granule has been formed, as shown in Fig. 5.

The following algorithm is a summary of the proposed higher type information granule formation procedure:

1. For each $\delta_{i, j}$ do:

2. Calculate $\mathrm{cv}=\frac{\sigma}{m}$,

3. Initialize $\sigma_{1,2}$ to $\sigma$,

4. Calculate $\mathrm{FOU}_{0}$ based on $\sigma_{1,2}$ and $m$,

5. Search until $\mathrm{cv}==\mathrm{FOU}_{k}$,

6. Increment $\sigma_{1,2}$ by $\pm \lambda$,

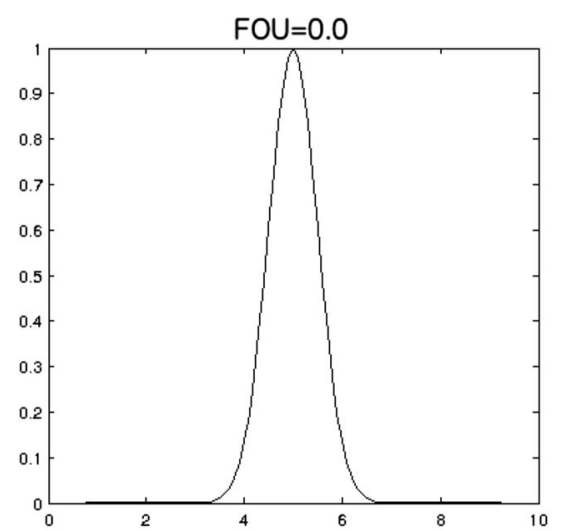

(a)

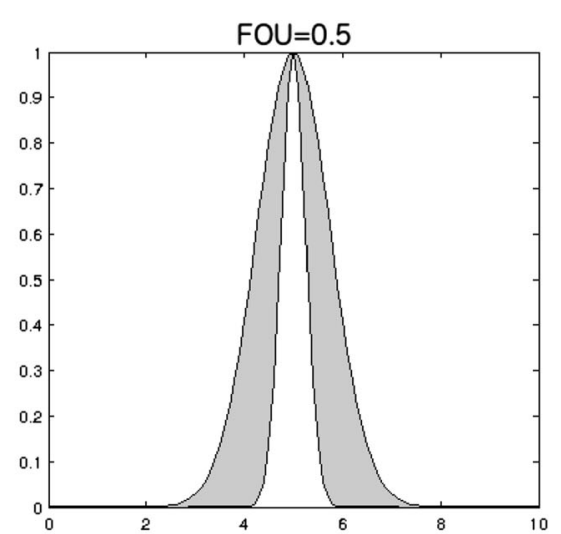

(b)

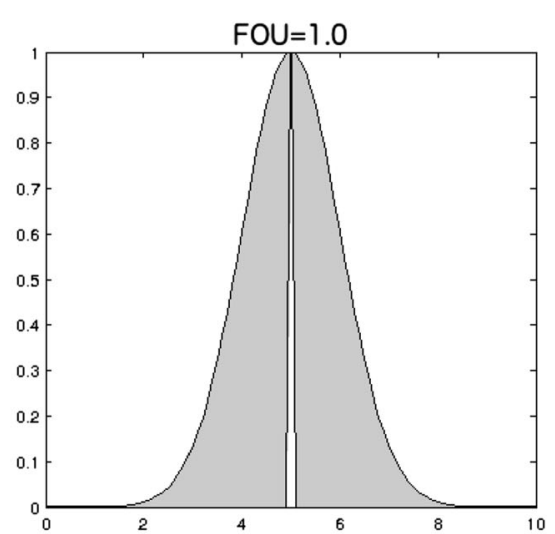

(c)

Fig. 4 Examples of varying degrees of FOU, where a FOU $=0, \mathbf{b}$ FOU $=0.5$, and $\mathbf{c}$ FOU $=1$ 
7. Update $\mathrm{FOU}_{k+1}$ based on $\sigma_{1,2}$ and $m$,

8. If $\left|\sigma_{1}, \sigma_{2}\right| \leq 2 \sigma$,

9. Return $\left\{\sigma_{1}=\varepsilon, \sigma_{2}=2 \sigma^{0}\right\}_{i, j}$,

10. Return $\left\{\sigma_{1}, \sigma_{2}\right\}_{i, j}$,

where $\sigma$ and $m$ are the initial value obtained from $\delta_{i, j}$ from the $i$-th rule and $j$-th input variable, $\varepsilon$ is a very small value, and $0 \leq \mathrm{FOU}_{k} \leq 1$.

\subsection{IT2 Sugeno fuzzy consequents via a granular- inspired steepest descent algorithm}

The second part of the proposed granular algorithm forms the consequents of the IT2 FIS. It is based on an existing algorithm used for optimizing an interval type-2 fuzzy neural network (IT2 FNN) (Castillo et al. 2009) where only the section for optimizing the consequents is used for the purpose of the proposed granular algorithm. With the addition of a user criterion, which controls the amount of uncertainty that affects the output interval, this algorithm now becomes granular in nature, in both antecedents and consequents.

The following optimization is focused only on consequents which are IT2 Sugeno polynomials in the form of Eq. (4), where $\left[g_{l}^{k}, g_{r}^{k}\right]$ are the left and right interval values of the $k$-th rule, $c_{k, i}$ is the coefficient of the $k$-th rule and $i$-th input, $x_{i}$ is the $i$-th input, $s_{k, i}$ is the spread on the $k$-th rule and $i$-th input, and $c_{k, 0}$ as well as $s_{k, 0}$ are independent constants for the coefficients and spreads, respectively.

$$
\begin{aligned}
& g_{l}^{k}=\sum_{i=1}^{n} c_{k, i} x_{i}+c_{k, 0}-\sum_{i=1}^{n} s_{k, i}\left|x_{i}\right|-s_{k, 0}, \\
& g_{r}^{k}=\sum_{i=1}^{n} c_{k, i} x_{i}+c_{k, 0}+\sum_{i=1}^{n} s_{k, i}\left|x_{i}\right|+s_{k, 0} .
\end{aligned}
$$

As the learning algorithm is a hybrid IT2 FNN, the feedforward section is first described by the following algorithm:

Step 1 Prepare inputs $X$ and outputs $Y$, shown in Eq. (5), with $i$ inputs, $j$ outputs, $p$ data pairs, $n$ number of inputs, $m$ number of outputs, and $q$ data pairs.

$$
\begin{aligned}
& X=\left[x_{i, p}\right] \quad \forall i=1, \ldots, n \quad \text { and } \quad p=1, \ldots, q, \\
& Y=\left[y_{j, p}\right] \quad \forall j=1, \ldots, m \quad \text { and } \quad p=1, \ldots, q .
\end{aligned}
$$

Step 2 Calculate individual firing strengths for all membership functions, IT2 Gaussian membership functions with uncertainty in the standard deviation (IT2gaussianMF), shown in Eq. (6), where $\mu_{\tilde{F}_{i}^{k}}\left(x_{i}^{\prime}\right)$ is the IT2 FS for the $k$-th rule and $i$-th input, $\underline{\mu}_{\tilde{F}_{i}^{k}}\left(x_{i}^{\prime}\right)$ and $\bar{\mu}_{\tilde{F}_{i}^{k}}$ are the lower and upper membership functions, respectively, as shown in Eq. (7), ${ }^{1} \sigma_{k, i}$ and ${ }^{2} \sigma_{k, i}$ are the first and second standard deviation of the $k$-th rule and $i$-th input, $m_{k . i}$ is the mean (center) of the $k$-th rule and $i$-th input, and $x^{\prime}$ is the current input value.

$$
\begin{aligned}
\mu_{\tilde{F}_{i}^{k}}\left(x_{i}^{\prime}\right) & =\left[\underline{\mu}_{\tilde{F}_{i}^{k}}\left(x_{i}^{\prime}\right), \bar{\mu}_{\tilde{F}_{i}^{k}}\left(x_{i}^{\prime}\right)\right] \\
& =\operatorname{IT} 2 \operatorname{gaussianMF}\left(x_{i}^{\prime},{ }^{1} \sigma_{k, i},{ }^{2} \sigma_{k, i}, m_{k . i}\right),
\end{aligned}
$$

$\underline{\mu}_{\tilde{F}_{i}^{k}}\left(x_{i}^{\prime}\right)=\exp \left[-\frac{1}{2}\left(\frac{x_{i}^{\prime}-m_{k, i}}{1 \sigma_{k, i}}\right)^{2}\right]$,

$\bar{\mu}_{\tilde{F}_{i}^{k}}\left(x_{i}^{\prime}\right)=\exp \left[-\frac{1}{2}\left(\frac{x_{i}^{\prime}-m_{k, i}}{{ }^{2} \sigma_{k, i}}\right)^{2}\right]$.

Step 3 Calculate all firing sets, shown in Eq. (8), where $\underline{\alpha}^{k}$ and $\bar{\alpha}^{k}$ are the lower and upper firing strengths for the $k$-th rule.

$$
\begin{aligned}
\underline{\alpha}^{k} & =\prod_{i=1}^{n} \underline{\mu}_{\tilde{F}_{i}^{k}}\left(x_{i}^{\prime}\right)=\prod_{i=1}^{n} \exp \left[-\frac{1}{2}\left(\frac{x_{i}^{\prime}-m_{k, i}}{{ }^{1} \sigma_{k, i}}\right)^{2}\right] \\
& =\exp \left[-\frac{1}{2} \sum_{i=1}^{n}\left(\frac{x_{i}^{\prime}-m_{k, i}}{{ }^{1} \sigma_{k, i}}\right)^{2}\right], \\
\bar{\alpha}^{k} & =\prod_{i=1}^{n} \bar{\mu}_{\tilde{F}_{i}^{k}}\left(x_{i}^{\prime}\right)=\prod_{i=1}^{n} \exp \left[-\frac{1}{2}\left(\frac{x_{i}^{\prime}-m_{k, i}}{{ }^{2} \sigma_{k, i}}\right)^{2}\right] \\
& =\exp \left[-\frac{1}{2} \sum_{i=1}^{n}\left(\frac{x_{i}^{\prime}-m_{k, i}}{2 \sigma_{k, i}}\right)^{2}\right] .
\end{aligned}
$$

Step 4 Calculate all rule consequents, shown in Eq. (9), where ${ }^{\text {left }} g_{j}^{k}\left(x^{\prime}\right)$ and right $g_{j}^{k}\left(x^{\prime}\right)$ are the left and right side of the consequent interval on the $k$-th rule and $j$-th output.

$$
\begin{aligned}
\text { left } g_{j}^{k}\left(x^{\prime}\right)= & \sum_{i=1}^{n} c_{k, i}^{j} x_{i}^{\prime}+c_{k, 0}^{j} \\
& -\sum_{i=1}^{n} s_{k, i}^{j}\left|x_{i}^{\prime}\right|-s_{k, 0}^{j}, \\
\text { right } g_{j}^{k}\left(x^{\prime}\right)= & \sum_{i=1}^{n} c_{k, i}^{j} x_{i}^{\prime}+c_{k, 0}^{j} \\
& +\sum_{i=1}^{n} s_{k, i}^{j}\left|x_{i}^{\prime}\right|+s_{k, 0}^{j} .
\end{aligned}
$$

Step 5 Calculate type-reduction via Karnik-Mendel (KM) algorithm (Mendel 2009), shown in Eqs. (10)(13), where $\hat{y}_{j}^{\text {left }}\left(x^{\prime}\right)$ and $\hat{y}_{j}^{\text {right }}\left(x^{\prime}\right)$ are the final output interval values, left and right, respectively, on the $j$-th output, $\underline{\alpha}$ and $\bar{\alpha}$ are the lower and upper firing strengths, $x^{\prime}$ is the current input value, $\omega_{k}^{\text {left }}$ and $\omega_{k}^{\text {right }}$ are the optimized firing strengths, left and right (obtained through the KM algorithm).

$\left[\hat{y}_{j}^{\text {lef } t}, \omega^{\text {left }}\right]=\mathrm{KM}\left({ }^{\text {left }} g_{j}^{k}, \underline{\alpha}, \bar{\alpha}\right)$, 


$$
\begin{aligned}
& \hat{y}_{j}^{\text {left }}\left(x^{\prime}\right)=\frac{\sum_{k=1}^{r} \omega_{k}^{\text {lef } t} \cdot{ }^{\text {lef } t} g_{j}^{k}}{\sum_{k=1}^{r} \omega_{k}^{\text {left }}} \\
& =\frac{\sum_{k=1}^{L} \bar{\alpha}_{u}^{i_{u}^{\text {left }}(k)} \cdot{ }^{\text {left }} g_{j}^{i_{u}^{\text {left }}(k)}+\sum_{k=L+1}^{r} \underline{\alpha}_{\ell}^{\dot{\alpha}_{\ell}^{\text {left }}(k)} \cdot{ }^{\text {left }} g_{j}^{j_{\ell}^{\text {left }}(k)}}{\sum_{k=1}^{L} \bar{\alpha}_{u}^{i_{u}^{\text {left }}(k)}+\sum_{k=L+1}^{r} \underline{\alpha}_{\ell}^{i^{\text {left }}(k)}}, \\
& {\left[\hat{y}_{j}^{\text {right }}, \omega^{\text {right }}\right]=\mathrm{KM}\left({ }^{\text {right }} g_{j}^{k}, \underline{\alpha}, \bar{\alpha}\right) \text {, }} \\
& \hat{y}_{j}^{\text {right }}\left(x^{\prime}\right)=\frac{\sum_{k=1}^{r} \omega_{k}^{\text {right }} \cdot \text { right } g_{j}^{k}}{\sum_{k=1}^{r} \omega_{k}^{\text {right }}}
\end{aligned}
$$

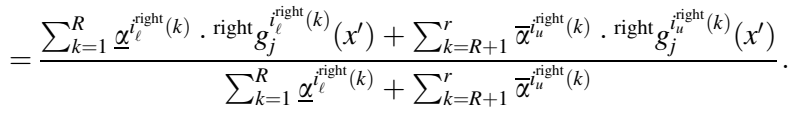

Step 6 Defuzzify interval $\left[\hat{y}_{j}^{\text {left }}\left(x^{\prime}\right), \hat{y}_{j}^{\text {right }}\left(x^{\prime}\right)\right]$, shown in Eq. (14), where $\hat{y}_{j}\left(x^{\prime}\right)$ is a crisp value.

$\hat{y}_{j}\left(x^{\prime}\right)=\frac{\hat{y}_{j}^{\mathrm{left}}\left(x^{\prime}\right)+\hat{y}_{j}^{\mathrm{right}}\left(x^{\prime}\right)}{2}$.

Step 7 Calculate the error function $e^{\rho}$, which is the output difference error, shown in Eq. (15). It is used for adjusting $c_{k, j}^{j}$ and $c_{k, 0}^{j}$, where $y_{j, p}$ is the obtained output, $\hat{y}_{j, p}$ is the reference output, $e_{j, p}$ is the error for the $j$-th output and $p$-th data pair.

$e_{j, p}^{\rho}=y_{j, p}-\hat{y}_{j, p}$

Step 8 Calculate the error function $e^{v}$, which is the output coverage error. It is used for adjusting $s_{k, i}^{j}$ and $s_{k, 0}^{j}$, where $m$ is the number of outputs of the IT2 FIS. The following algorithm describes how this error is obtained.

1. Scale $=100 / \mathrm{m}$,

2. Count $=0$,

3. If left $\hat{y}_{j, p} \leq y_{j, p} \leq{ }^{\text {right }} \hat{y}_{j, p}$ then,

4. Increase count by 1 ,

5. $e_{p}^{v}=100-\frac{\text { count } * \text { scale }}{m}$.

Step 9 Calculate the error function $e^{\tau}$, which is the output uncertainty width. It is used for controlling the final interval size of each output's uncertainty, where $\mathrm{FOU}_{j, p}$ is the footprint of uncertainty on the $j$-th output and $p$-th data pair, range ${ }_{j}^{k}$ is the difference between the ranges [left $\left.g_{j}^{k}\left(x^{\prime}\right),{ }^{\text {right }} g_{j}^{k}\left(x^{\prime}\right)\right]$ of the universe of discourse on the $j$-th output and $k$-th rule, and $m$ is the number of outputs of the IT2 FIS. The following algorithm describes how this error is obtained.

1. $\mathrm{FOU}_{j, p}={ }^{\text {right }} \hat{y}_{j, p}-{ }^{\text {left }} \hat{y}_{j, p}$.

2. range $_{j}^{k}={ }^{\text {right }} g_{j}^{k}\left(x^{\prime}\right)-{ }^{\text {left }} g_{j}^{k}\left(x^{\prime}\right)$.
3. $e_{p}^{\tau}=\frac{\frac{100}{\text { range }_{j}^{k}} * \operatorname{mean}(\mathrm{FOU})}{m}$.

Step 10 Calculate the composite error $e_{p}^{\varphi}$. It is used for adjusting the multiobjective function of the granular output interval, shown in Eq. (16), where $\beta$ is a scaling user criterion used for controlling the size of the output interval.

$e_{p}^{\varphi}=\beta * \frac{\left(e_{p}^{v}+e_{p}^{\tau}\right)}{2}$

In the feedforward algorithm above, steps 1-6, follow the normal process in which an IT2 Takagi-Sugeno-Kang fuzzy logic system (IT2 TSK FLS) is inferred, whereas steps 7-10 are used for calculating various errors required for the proposed granular adjustment of IT2 TSK consequents. Regarding the error $e^{\rho}$, it will be used as a standalone error for adjusting the parameters of $c$, while $e^{\varphi}$ will be used solely for adjusting the parameters of $s$. The backpropagation procedure is where the modifications were made on the original algorithm, which is shown by the following procedure.

Given a training pair $\{X: Y\}_{p}^{q}=1$, to calculate the IT2 TSK consequent parameters, error functions $\left\{e^{\rho}, e^{\varphi}\right\}$ must be minimized, and a steepest descent algorithm is used for this purpose.

Before continuing, a note must be taken on the addition of granular IT2 TSK outputs. With a user control criterion which has been added that adjusts the size of the output information granule, i.e., a means to specify the desired coverage by the output. Call this user control criterion $\beta$, where $\beta \geq 0$. As $\beta$ decreases into a near zero value, the output coverage also decreases, where instead of having an interval a single value would be obtained, such that it would be a defuzzified output; when $\beta$ increases so does the output interval. Although more coverage is good, too much coverage over-generalizes the model.

Four distinct steepest descent algorithms are used, each one for a different purpose. Equations (17), (18) are used for modifying the values of parameters $\left\{c_{k, i}^{j}, c_{k, 0}^{j}\right\}$, where Eq. (17) adjusts the dependent parameters, and Eq. (18) adjusts the independent parameters. Just as well, Eqs. (19), (20) are used for modifying the values of parameters $\left\{s_{k, i}^{j}, s_{k, 0}^{j}\right\}$, where Eq. (19) adjusts the dependent parameters, and Eq. (20) adjusts the independent parameters.

$$
{ }^{n e w} c_{k, i}^{j}={ }^{\text {old }} c_{k, i}^{j}+\eta \cdot \frac{1}{2} \cdot e_{p}^{\rho} \cdot\left[\frac{\omega_{k}^{\text {right }}}{\sum_{k=1}^{r} \omega_{k}^{\text {right }}}+\frac{\omega_{k}^{\text {left }}}{\sum_{k=1}^{r} \omega_{k}^{\ell e f t}}\right] \cdot x_{i},
$$




$$
{ }^{n e w} c_{k, 0}^{j}={ }^{\text {old }} c_{k, 0}^{j}+\eta \cdot \frac{1}{2} \cdot e_{p}^{\rho} \cdot\left[\frac{\omega_{k}^{\text {right }}}{\sum_{k=1}^{r} \omega_{k}^{\text {right }}}+\frac{\omega_{k}^{\text {left }}}{\sum_{k=1}^{r} \omega_{k}^{\text {left }}}\right] \text {, }
$$$$
\begin{aligned}
{ }^{n e w} s_{k, i}^{j}= & { }^{\text {old }} s_{k, i}^{j}+\eta \cdot \frac{1}{2} \cdot e_{p}^{\varphi} \cdot\left[\frac{\omega_{k}^{r i g h t}}{\sum_{k=1}^{r} \omega_{k}^{r i g h t}}-\frac{\omega_{k}^{\text {left }}}{\sum_{k=1}^{r} \omega_{k}^{\text {left }}}\right] \\
& \cdot\left|x_{i}\right|,
\end{aligned}
$$

$$
{ }^{n e w} s_{k, 0}^{j}={ }^{\text {old }} s_{k, 0}^{j}+\eta \cdot \frac{1}{2} \cdot e_{p}^{\varphi} \cdot\left[\frac{\omega_{k}^{r i g h t}}{\sum_{k=1}^{r} \omega_{k}^{r i g h t}}-\frac{\omega_{k}^{\text {left }}}{\sum_{k=1}^{r} \omega_{k}^{\text {left }}}\right] .
$$

Here, $\eta$ is a learning rate. This parameter was changed dynamically (Jang 1993) via a simple heuristic:

1. If the objective function experiences four consecutive reductions, then increase $\eta$ by a small percentage.

2. If the objective function experiences four consecutive combinations of one increase and one reduction, then decrease $\eta$ by a small percentage.

\section{Experimental results and discussion}

Some experimentation was done with various datasets so as to evaluate the performance of the proposed granular model. Two synthetic datasets are used; a composite curve (The MathWorks, Inc., Natick, Massachusetts 2013), with one input ( $x$ ), one output $(y)$, and having 94 data pairs; and Mackey-Glass (Mackey and Glass 1977), with one input $(x)$, one output $(y)$, and having 1200 data pairs. And two real datasets; air passengers (Hyndman 2015), with one input (month), one output (air passenger frequency), and having 144 data pairs; and thermex behavior (The MathWorks, Inc., Natick, Massachusetts 2013), with one input (temperatures in degrees Kelvin), one output (coefficient of thermal expansion for copper), and 236 data pairs. Their graphical behavior can be found in Fig. 6 .

To measure performance of granular outputs from the formed Higher Type granular models, the coverage of the outputs as well as the coefficient of determination $\left(R^{2}\right)$ are measured. The testing method verifies that the interval output of the IT2 FIS had good coverage of the reference targets, while at the same time having good modeling performance.

Two sets of experiments were carried out. The first experimental set was performed on three datasets as shown in Table 1 , where $R^{2}$ and output coverage measure the performance of the proposed algorithm with curve fitting. For these experiments, 30 runs were executed to obtain the mean and standard deviation (std) for both measures. For the case of the composite curve, its parameters are: 9 rules, $\eta=0.002$, and $\beta=0.1$. For the thermex behavior, its parameters are: 12 rules, $\eta=0.000001$, and $\beta=2$. And for the air passengers dataset, its parameters are: 25 rules, $\eta=0.0001$, and $\beta=3$.

These curve fitting experiments show that the proposed granular modeling scheme is fairly stable as the standard deviation for $R^{2}$ is very low; although the stability of the output coverage is not as good, it is still somewhat low. As for the acquired mean values, $R^{2}$ was measured with respect to the test targets. As such, both results for composite curve and repetitive curve obtained good results, as their $R^{2}$ was above 0.9 ; but for the air passengers results, its obtained value is not as good, yet it obtained an acceptable value of 0.829708 . As for the obtained values of the output coverage, the air passengers experiment obtained the highest value of $R^{2}$, followed by the result of the repetitive curve; finally, the value of $R^{2}$ for the composite curve is not very good, such that a mean value of $73.07 \%$ coverage was obtained.

The second experimental set was performed on two datasets as shown in Table 2, where $R^{2}$ and output coverage measure performance on forecasting datasets. For these experiments, $50 \%$ training and $50 \%$ test was done; and only one test run was executed. For the case of MackeyGlass, its parameters were set to: 10 rules, $\eta=0.00001$, and $\beta=1.5$. And for air passengers dataset, set parameters were: 15 rules, $\eta=0.00001$, and $\beta=1.5$.

These experiments show that the proposed granular model has fairly good forecasting capabilities, shown through the obtained values of $R^{2}$. As for the output coverage measure, it obtained great results, achieving $100 \%$ coverage of all test target values.

\section{Conclusions}

This paper presented an algorithm which forms granular models with higher type information granules, via IT2 FS representation. Based on the dispersion of data, higher type information granules are formed as the antecedents of an IT2 FIS, followed by a granular-inspired learning algorithm which adjusts the consequent parameters for an IT2 TSK FIS.

When the antecedents are formed, not all information granules are higher type, some are also non-higher type; this gives a better representation of the information since a combination of higher and non-higher type information granules gives a better representation of the final granular model. Having IT2 TSK consequents, the granular-inspired learning algorithm requires a user criterion which ultimately controls the final size of the output interval; this is achieved by a multiobjective optimization error function 

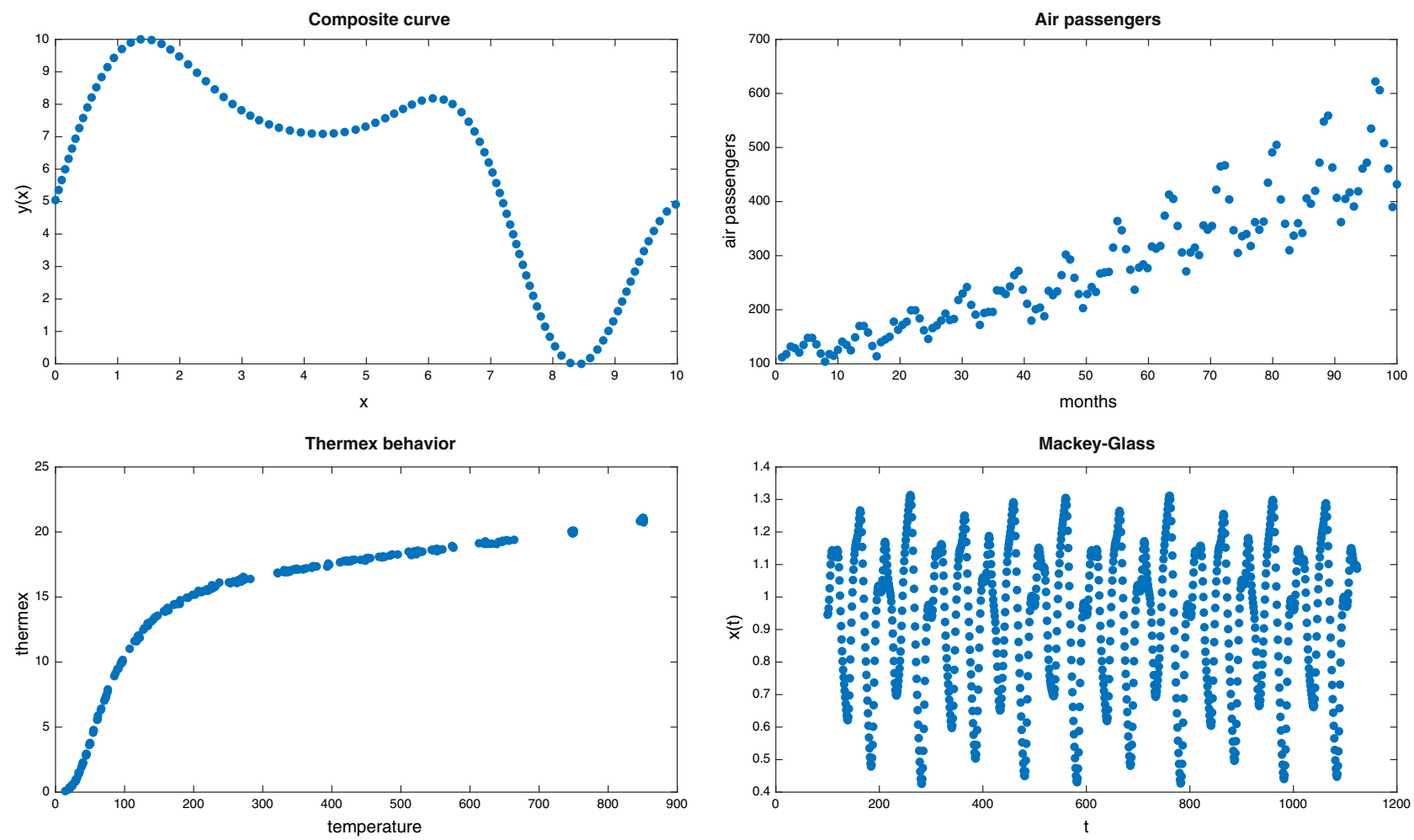

Fig. 6 Graphical behavior of the experimental sets chosen to test the performance of the proposed granular modeling algorithm

Table 1 Results for curve fitting experiments, where $R^{2}$ and output coverage are measured

\begin{tabular}{lllllr}
\hline Dataset & $R^{2}$ & & \multicolumn{2}{l}{ Output coverage } \\
\cline { 2 - 3 } & Mean & Std & & Mean & \multicolumn{1}{c}{ Std } \\
\hline Composite curve & 0.926152 & 0.33368 & & 73.070175 & 11.802886 \\
Thermex behavior & 0.952514 & 0.035048 & & 81.950355 & 5.955896 \\
Air passengers & 0.829708 & 0.303469 & & 91.83908 & 3.759262 \\
\hline
\end{tabular}

Table 2 Results for forecasting experiments, where, $R^{2}$ and output coverage are measured

\begin{tabular}{lll}
\hline Dataset & $R^{2}$ & Output coverage \\
\hline Mackey-Glass & 0.964739 & 100 \\
Air passengers & 0.894817 & 100 \\
\hline
\end{tabular}

which divides the process into two: an optimization for centering the general behavior of the output with respect to the reference targets, and an optimization for controlling the coverage by the output interval. An additional point in favor of this learning algorithm is its speed; this learning algorithm executes in a few minutes rather than hours in comparison with other optimization or evolutionary algorithms.
Included experimentation evaluates the proposed granular modeling algorithm in the sense of measuring how well can it perform with respect to curve fitting and data forecasting.

\section{References}

Atanassov KT (1986) Intuitionistic fuzzy sets. Fuzzy Sets Syst 20:87-96. doi:10.1016/S0165-0114(86)80034-3

Bezdek JC, Ehrlich R, Full W (1984) FCM: the fuzzy c-means clustering algorithm. Comput Geosci 10:191-203. doi:10.1016/ 0098-3004(84)90020-7

Castillo O, Melin P (2012) Recent advances in interval type-2 fuzzy systems. Springer, Berlin

Castillo O, Melin P, Castro JR, Rodríguez-Díaz A (2009) A hybrid learning algorithm for a class of interval type-2 fuzzy neural networks. Inf Sci (Ny) 179:2175-2193. doi:10.1016/j.ins.2008. 10.016

Chen S-M, Chang Y-C (2011a) Fuzzy rule interpolation based on the ratio of fuzziness of interval type-2 fuzzy sets. Expert Syst Appl 38:12202-12213. doi:10.1016/j.eswa.2011.03.084

Chen S-M, Chang Y-C (2011b) Fuzzy rule interpolation based on principle membership functions and uncertainty grade functions of interval type-2 fuzzy sets. Expert Syst Appl 38:11573-11580. doi:10.1016/j.eswa.2011.03.035

Chen S-M, Hong J-A (2014) Fuzzy multiple attributes group decisionmaking based on ranking interval type-2 fuzzy sets and the TOPSIS method. IEEE Trans Syst Man Cybern Syst 44:1665-1673. doi:10.1109/TSMC.2014.2314724 
Chen S-M, Lee L-W (2010a) Fuzzy Multiple Criteria Hierarchical Group decision-making based on interval type-2 fuzzy sets. IEEE Trans Syst Man, Cybern Part A Syst Humans 40:1120-1128. doi:10.1109/TSMCA.2010.2044039

Chen S-M, Lee L-W (2010b) Fuzzy multiple attributes group decision-making based on the interval type-2 TOPSIS method. Expert Syst Appl 37:2790-2798. doi:10.1016/j.eswa.2009.09. 012

Chen S-M, Lee L-W (2010c) Fuzzy multiple attributes group decision-making based on the ranking values and the arithmetic operations of interval type-2 fuzzy sets. Expert Syst Appl 37:824-833. doi:10.1016/j.eswa.2009.06.094

Chen S-M, Lee L-W (2011) Fuzzy interpolative reasoning for sparse fuzzy rule-based systems based on interval type-2 fuzzy sets. Expert Syst Appl 38:9947-9957. doi:10.1016/j.eswa.2011.02. 035

Chen S-M, Wang C-Y (2013) Fuzzy decision making systems based on interval type-2 fuzzy sets. Inf Sci (Ny) 242:1-21. doi:10. 1016/j.ins.2013.04.005

Chen G, Ying M, Liu Y (2009) Dealing with uncertainty and fuzziness in intelligent systems. Int J Intell Syst 24:223-225. doi:10.1002/int.20333

Chen S-M, Yang M-W, Lee L-W, Yang S-W (2012) Fuzzy multiple attributes group decision-making based on ranking interval type2 fuzzy sets. Expert Syst Appl 39:5295-5308. doi:10.1016/j. eswa.2011.11.008

Chen S-M, Chang Y-C, Pan J-S (2013a) Fuzzy Rules interpolation for sparse fuzzy rule-based systems based on interval type-2 gaussian fuzzy sets and genetic algorithms. IEEE Trans Fuzzy Syst 21:412-425. doi:10.1109/TFUZZ.2012.2226942

Chen S-M, Lee L-W, Shen VRL (2013b) Weighted fuzzy interpolative reasoning systems based on interval type-2 fuzzy sets. Inf Sci (Ny) 248:15-30. doi:10.1016/j.ins.2013.05.002

Cheng S-H, Chen S-M, Huang Z-C (2016) Autocratic decision making using group recommendations based on ranking interval type-2 fuzzy sets. Inf Sci (Ny) 361:135-161. doi:10.1016/j.ins. 2016.04 .035

Hyndman RJ (2015) Time Series Data Library. http://data.is/ TSDLdemo. Accessed 20 Dec 2015

Jang JSR (1993) ANFIS: adaptive-network-based fuzzy inference system. IEEE Trans Syst man Cybern 23:665-685. doi:10.1109/ 21.256541

Jurado K, Ludvigson SC, Ng S (2015) Measuring uncertainty. Am Econ Rev 105:1177-1216. doi:10.1257/aer.20131193

Klir GJ (2005) Uncertainty and information: foundations of generalized information theory. Wiley-IEEE Press, New Jersey

Klir GJ, Wierman MJ (1999) Uncertainty-based information: elements of generalized information theory, 2nd edn. PhysicaVerlag Heidelberg, Heidelberg

Mackey MC, Glass L (1977) Oscillation and chaos in physiological control systems. Science 197:287-289

Mendel JM (2009) Enhanced Karnik-Mendel algorithms. IEEE Trans Fuzzy Syst 17:923-934. doi:10.1109/TFUZZ.2008.924329

Mendel JM (2016) A comparison of three approaches for estimating (synthesizing) an interval type-2 fuzzy set model of a linguistic term for computing with words. Granul Comput 1:59-69. doi:10. 1007/s41066-015-0009-7
Mo H, Wang F-Y, Zhou M et al (2014) Footprint of uncertainty for type-2 fuzzy sets. Inf Sci (Ny) 272:96-110. doi:10.1016/j.ins. 2014.02.092

Pawlak Z (1982) Rough sets. Int J Comput Inf Sci 11:341-356. doi:10.1007/BF01001956

Pedrycz W (2007) Granular computing - the emerging paradigm. J Uncertain Syst 1:38-61

Pedrycz W (2013) Information granules of higher type and higher order, and hybrid information granules. In: Granular computing. analysis and design of intelligent systems. CRC Press, Florida, pp 47-60

Pedrycz W, Chen SM (2011) Granular computing and intelligent systems: design with information granules of higher order and higher type. Springer, Berlin Heidelberg

Pedrycz W, Chen SM (2015a) Information granularity, big data, and computational intelligence, $1 \mathrm{st}$ edn. Springer International Publishing, New York

Pedrycz W, Chen SM (2015b) Granular Computing and DecisionMaking: Interactive and Iterative Approaches, 1st edn. Springer International Publishing, New York

Pedrycz W, Vukovich G (2002) Granular computing with shadowed sets. Int J Intell Syst 17:173-197. doi:10.1002/int.10015

Sai YSY, Nie PNP, Chu DCD (2005) A model of granular computing based on rough set theory. In: 2005 IEEE International Conference on Granular Computing. pp 233-236

Starczewski JT (2008) On defuzzification of interval type-2 fuzzy sets. Artificial intelligence and soft computing -ICAISC 2008. Springer, Berlin, pp 333-340

The MathWorks, Inc., Natick, Massachusetts US (2013) MATLAB Release 2013b

Vasilakos A, Stathakis D (2004) Granular neural networks for land use classification. Soft Comput 9:332-340. doi:10.1007/s00500004-0412-5

Weise K, Woger W (1993) A Bayesian theory of measurement uncertainty. Meas Sci Technol 4:1-11. doi:10.1088/0957-0233/ $4 / 1 / 001$

Ying H (2009) Interval Type-2 Takagi-Sugeno fuzzy systems with linear rule consequent are universal approximators. In: NAFIPS 2009-2009 Annual Meeting of the North American Fuzzy Information Processing Society. IEEE, pp 1-5

Yu X, Mehrotra S (2003) Capturing uncertainty in spatial queries over imprecise data. In: Mař́k V, Retschitzegger W, Štěpánková O (eds) Database and expert systems applications: proceedings of 14th International Conference DEXA 2003, Prague, Czech Republic, September 1-5, 2003. Springer, Berlin, Heidelberg, pp 192-201. doi:10.1007/978-3-540-45227-0_20

Zadeh LA (1965) Fuzzy Sets. Inf. Control 8:338-353

Zadeh LA (1975) The concept of a linguistic variable and its application to approximate reasoning-I. Inf Sci (Ny) 8:199-249

Zhang LZL, Zhang BZB (2005) Quotient space based multi-granular computing. In: 2005 IEEE International Conference on Granular Computing. p 98

Zhang YZY, Zhu XZX, Huang ZHZ (2009) Fuzzy sets based granular logics for granular computing. In: 2009 International Conference on Computational Intelligence and Software Engineering. IEEE, New York, pp 1-6 